\title{
ELASTIC WAVES WITH FINITE ROTATIONS
}

\author{
I. D. Kotchergenko
}

Kotchergenko Engenharia Ltda., Belo Horizonte, Brazil (koteng@terra.com.br)

\begin{abstract}
Waves are represented through the superposition of the four fundamental modes of the plane areolar strain theory. This theory was presented at references [10] to [13], with application to finite rotations, orthotropic materials and finite element models, but a summary review is here presented in order to make this paper self contained and also to disclose the areolar strain concept, which although divulged for two decades still faces with poor acceptance by the scientific community. It is shown that the areolar strain approach does not distinguish finite from infinitesimal strain due to the fact that in addition to the traditional "forward" strain it incorporates the "sidelong" strain into its imaginary part. Instead of comparing the change in distance between two contiguous points, the areolar strains presents the complete state of finite strains on an areola that surrounds a given point. Only first derivatives are used, as expected due to the physical meaning of strain and having in mind that the relative displacement between two arbitrary points of the plane should be obtained through a single line integration of the strain, along any path of integration joining these points. The areolar strain fulfils these conditions. Now, an approach for tackling with equivoluminal waves of finite rotation is presented, assuming that the gain of volume due the mathematical finite rotation is neutralized by the mathematical shrinkages due to the complex shear strain.
\end{abstract}

Keywords: Elastic waves, Finite rotations, Areolar strain, Complex shear.

\section{INTRODUCTION}

The development of the theory of two-dimensional elasticity hereof used is grounded on the concept of areolar strain. This concept was first presented by the writer $[11,12,13]$. The equilibrium equations for finite rotations were presented at Ref. [10]. A summary review of the areolar strain concept is provided in order to make this article self contained. In this approach, the strain is obtained by the division of two complex-valued quantities associated with $2 \mathrm{D}$ vectors. The areolar strain concept allows visualizing the state of strain on an areola that surrounds a given point. The real part of the areolar strain is a radial strain while the imaginary part is either a circumferential strain or a rotation, (see Fig.1). Under this concept there is no distinction between infinitesimal and finite strain, except for rotations and care should be taken to calculate the change of volume. The fundamentals of the method of complex variable applied to solution of elastic problems can be found on Refs. [4,7,9]. 


\section{THE AREOLAR STRAIN CONCEPT}

Let a region in the plane of the variables $z=x+i y$ and $\bar{z}=x-i y$ be mapped in a oneto-one manner onto the plane of the displacements $u(x, y)$ and $v(x, y)$ by means of the transformation $w(z, \bar{z})=u(x, y)+i v(x, y)$. The areolar strain is defined as the gradient of the vector field $w(z, \bar{z})$, through the Riemann derivative [14]:

$$
\varepsilon=\lim _{z \rightarrow z_{0}} \frac{w-w_{0}}{z-z_{0}}=\frac{\frac{\partial w}{\partial z} d z+\frac{\partial w}{\partial \bar{z}} d \bar{z}}{d z},
$$

or

$$
\varepsilon=\frac{\partial w}{\partial z}+\frac{\partial w}{\partial \bar{z}} e^{-i 2 \alpha}
$$

where the polar form has been used for the ratio

$$
\frac{d \bar{z}}{d z}=\frac{|d \bar{z}| e^{-i \alpha}}{|d z| e^{i \alpha}}=e^{-i 2 \alpha}
$$

The last expression presupposes that $z$ tends to $z_{0}$, maintaining the direction $\alpha$. Equation (2) is obtained making

$$
\begin{aligned}
& d u=\frac{\partial u}{\partial x} d x+\frac{\partial u}{\partial y} d y, d v=\frac{\partial v}{d x} d x+\frac{\partial v}{d y} d y, \\
& d u+i d v=\frac{\partial w}{\partial z}(d x+i d y)+\frac{\partial w}{\partial \bar{z}}(d x-i d y),
\end{aligned}
$$

with

$$
\begin{aligned}
& 2 \frac{\partial w}{\partial z}=\left(\frac{\partial u}{\partial x}+\frac{\partial v}{\partial y}\right)+i\left(\frac{\partial v}{\partial x}-\frac{\partial u}{\partial y}\right)=\theta+i 2 \omega \\
& 2 \frac{\partial w}{\partial \bar{z}}=\left(\frac{\partial u}{\partial x}-\frac{\partial v}{\partial y}\right)+i\left(\frac{\partial v}{\partial x}+\frac{\partial u}{\partial y}\right)=\xi+i \gamma
\end{aligned}
$$

Equations (5) and (6) are the Kolosov-Wirtinger derivatives [14]. The areolar strain can hence be written in the form

$$
\varepsilon=1 / 2(\theta+i 2 \omega)+1 / 2(\xi+i \gamma) e^{-2 i \alpha} .
$$

When viewed in the polar form, see Fig. 1, the real part of the areolar strain represents a radial strain and the imaginary part represents either, a circumferential strain or a rotation. In other words, the linear part represents the forward strain, which correspond to the classical linear strain and the imaginary part represents a sidelong strain, which in the traditional approach is represented by the second order derivatives. The second complex term is the complex shear strain. 

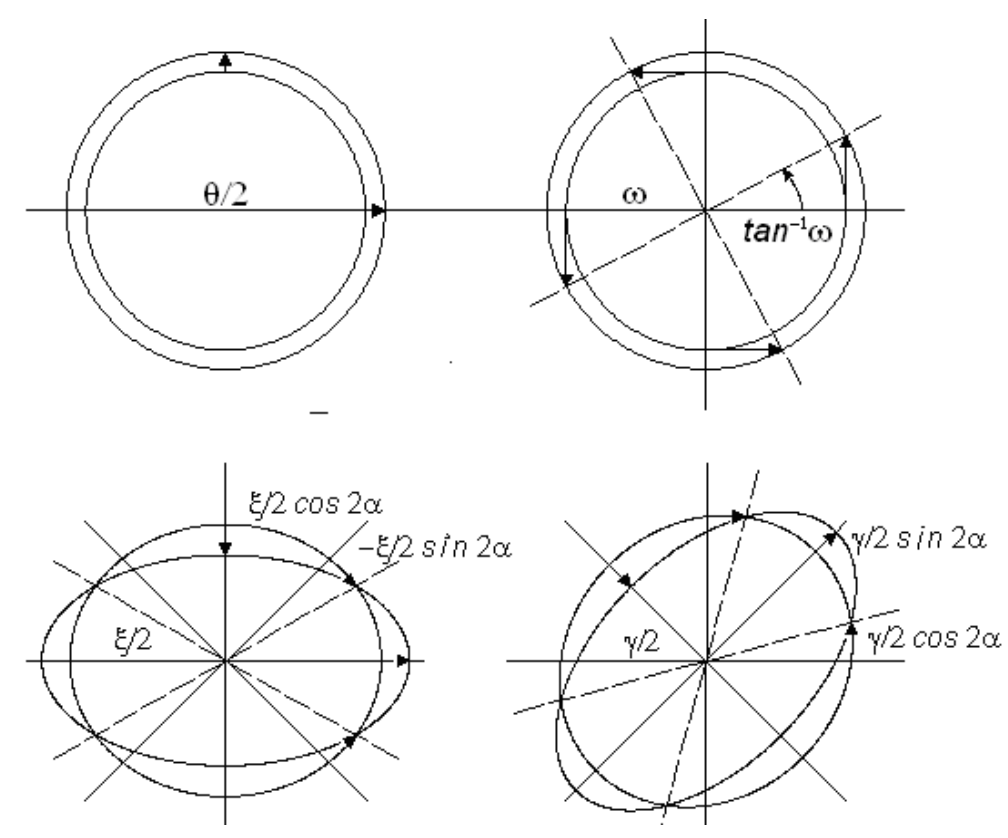

Figure 1. The four fundamental modes of the plane strain.

The components of the areolar strain are orthogonal as the quadratic form contained in the integrand of the work expression $U=1 / 2 \int C_{i j} \varepsilon_{i} \varepsilon_{j} d V$, can be converted into its canonic form

$$
U=1 / 2 \int\left[1 / 2\left(C_{11}+C_{12}\right) \theta^{2}+1 / 2\left(C_{11}-C_{12}\right) \xi^{2}+C_{13} \gamma^{2}\right] d V
$$

\subsection{Compatibility Equations}

If $z_{0}$ and $z$ are two points pertaining to the complex plane, their relative displacement is given by

$$
w-w_{0}=\int_{C} \varepsilon d z=\int_{C}\left(\frac{\partial w}{\partial z}+\frac{\partial w}{\partial \bar{z}} \frac{d \bar{z}}{d z}\right) d z=\int_{C} \frac{\partial w}{\partial z} d z+\frac{\partial w}{\partial \bar{z}} d \bar{z}
$$

Since $w-w_{0}$ is independent of the path of integration $C$,

$$
d w=\frac{\partial w}{\partial z} d z+\frac{\partial w}{\partial \bar{z}} d \bar{z}
$$

is a total differential. Consequentially, the displacement field must comply with the condition of continuity

$$
\frac{\partial}{\partial \bar{z}}\left(\frac{\partial w}{\partial z}\right)-\frac{\partial}{\partial z}\left(\frac{\partial w}{\partial \bar{z}}\right)=0
$$


Separating the real and imaginary parts of this equation, the following compatibility equations are obtained:

$$
\begin{aligned}
& \frac{\partial}{\partial x}(2 \omega-\gamma)+\frac{\partial}{\partial y}(\theta+\xi)=0 \\
& \frac{\partial}{\partial y}(2 \omega+\gamma)-\frac{\partial}{\partial x}(\theta-\xi)=0 .
\end{aligned}
$$

Saint-Venant's compatibility equation is obtained from these equations through elimination of the mode $\omega$, by applying a cross-differentiation followed by a subtraction. SaintVenant's compatibility equation will then be satisfied for any field of rotations $\omega$, which may thus violate the compatibility conditions established by Eqs. (12) and (13). Saint-Venant's mistake was to assume $\omega$ as a rigid-body rotation instead of a field of rotations.

\subsection{Equilibrium Equations}

For isotropic material, $1 / 2\left(C_{11}+C_{12}\right)=\lambda+\mu, 1 / 2\left(C_{11}-C_{12}\right)=\mu$ and $C_{13}=\mu$. Thus, the work expression given by Eq. (8) reduces to

$$
U=\frac{1}{2} \int\left[(\lambda+\mu) \theta^{2}+\mu\left(\xi^{2}+\gamma^{2}\right)\right] d V
$$

where $\lambda$ and $\mu$ are Lame's elastic material constants for the plane strain state. The Euler equations for this functional are

$$
\begin{aligned}
& (\lambda+\mu) \frac{\partial \theta}{\partial x}+\mu\left(\frac{\partial \xi}{\partial x}+\frac{\partial \gamma}{\partial y}\right)=0 \\
& (\lambda+\mu) \frac{\partial \theta}{\partial y}+\mu\left(\frac{\partial \gamma}{\partial x}-\frac{\partial \xi}{\partial y}\right)=0
\end{aligned} .
$$

Taking into account that

$$
2 \frac{\partial}{\partial z}(\xi+i \gamma)=\left(\frac{\partial \xi}{\partial x}+\frac{\partial \gamma}{\partial y}\right)+i\left(\frac{\partial \gamma}{\partial x}-\frac{\partial \xi}{\partial y}\right)=\nabla^{2} u(x, y)+i \nabla^{2} v(x, y)
$$

where the symbol $\nabla^{2}$ stands for the Laplacian operator, Lamé's homogeneous equilibrium equations can be presented in the following complex form:

$$
2 \frac{\partial}{\partial \bar{z}}[(\lambda+\mu) \theta]+2 \frac{\partial}{\partial z}[\mu(\xi+i \gamma)]=0
$$

\subsection{Boundary Conditions}

Applying Green's formula in the complex form [2], 


$$
2 \iint_{\Omega} \frac{\partial}{\partial \bar{z}}[(\lambda+\mu) \theta]+\frac{\partial}{\partial z}[\mu(\xi+i \gamma)] d x d y=\frac{1}{i} \oint_{C}\left[(\lambda+\mu) \theta-\mu(\xi+i \gamma) \frac{d \bar{z}}{d z}\right] d z
$$

and the traction vector on boundary $\mathrm{C}$ results

$$
T=(\lambda+\mu) \theta-\mu(\xi+i \gamma) e^{-2 i \alpha}
$$

where $\alpha$ points towards the tangent to the contour curve $C$. Observe that if $n$ is the unit outward vector, normal to the element of arc $d s=|d z|$, then $n d s=-i d z=1 / i d z$. The real part of $T$ is the normal stress while the imaginary part of $T$ is the shear stress pointing at a direction rotated $\pi / 2$ counterclockwise with respect to the normal stress. The real part of $T$ is hence normal to the curve $\mathrm{C}$. The compatibility equations (12) and (13) can be rewritten in the following complex form

$$
\frac{\partial}{\partial \bar{z}}(\theta+2 i \omega)-\frac{\partial}{\partial z}(\xi+i \gamma)=0
$$

\section{PLANE WAVES IN INFINITE MEDIA}

Plane waves in homogeneous infinite media are shortly commented, using the approach of superimposing the fundamental strain modes shown in Fig 1.The addition of the inertial term to Lame's Eq. (16), gives

$$
(\lambda+\mu) 2 \frac{\partial \theta}{\partial \bar{z}}+\mu 2 \frac{\partial}{\partial z}(\xi+i \gamma)-\rho \frac{\partial^{2}(u+i v)}{\partial t^{2}}=0
$$

Substitution of the second term of the equilibrium equation above by the second term of the compatibility Eq. (19), gives the equilibrium equation in the form

$$
(\lambda+2 \mu) 2 \frac{\partial \theta}{\partial \bar{z}}+i \mu 4 \frac{\partial \omega}{\partial \bar{z}}-\rho \frac{\partial^{2}(u+i v)}{\partial t^{2}}=0
$$

Derivative in $\mathrm{z}$ results

$$
(\lambda+2 \mu) 2 \frac{\partial^{2} \theta}{\partial z \partial \bar{z}}+i \mu 4 \frac{\partial^{2} \omega}{\partial z \partial \bar{z}}-\rho \frac{\partial^{2}}{\partial t^{2}} \frac{\partial w}{\partial z} .
$$

As $4 \frac{\partial^{2}}{\partial z \partial \bar{z}}=\nabla^{2}$ and $\frac{\partial w}{\partial z}=\frac{1}{2}(\theta+i 2 \omega)$, separating the real and imaginary parts, we obtain the irrotational and the equivoluminal wave equations

$$
\begin{gathered}
\nabla^{2} \theta-\frac{\rho}{\lambda+2 \mu} \frac{\partial^{2} \theta}{\partial t^{2}}=0 \\
\nabla^{2} \omega-\frac{\rho}{\mu} \frac{\partial^{2} \omega}{\partial t^{2}}=0 .
\end{gathered}
$$

As the two other fundamental modes $\xi$ and $\gamma$ must vibrate together with either mode $\theta$ or mode $\omega$, in order to comply with the compatibility equation, similar wave 
equations governs those modes. It is easy to prove that by taking from the compatibility equation

$$
\frac{\partial \theta}{\partial \bar{z}}=\frac{\partial}{\partial z}(\xi+i \gamma)-2 \frac{\partial \omega}{\partial \bar{z}}
$$

And after grafting $\omega=0$ for the irrotational wave case, the substitution of $\frac{\partial \theta}{\partial \bar{z}}$ into the equilibrium equation gives

$$
(\lambda+2 \mu) 2 \frac{\partial}{\partial z}(\xi+i \gamma)-\rho \frac{\partial^{2} w}{\partial t^{2}}=0
$$

Taking the derivative in $\bar{z}$, as $4 \frac{\partial^{2}}{\partial z \partial \bar{z}}=\nabla^{2}$ and $\frac{\partial w}{\partial \bar{z}}=\frac{1}{2}(\xi+i \gamma)$, results

$$
\begin{aligned}
& \nabla^{2} \xi_{\theta}-\frac{\rho}{\lambda+2 \mu} \frac{\partial^{2} \xi_{\theta}}{\partial t^{2}}=0 \\
& \nabla^{2} \gamma_{\theta}-\frac{\rho}{\lambda+2 \mu} \frac{\partial^{2} \gamma_{\theta}}{\partial t^{2}}=0
\end{aligned}
$$

Now starting with the condition $\theta=0$ for the equivoluminal wave and following the same procedures, we get

$$
\begin{aligned}
& \nabla^{2} \xi_{\omega}-\frac{\rho}{\mu} \frac{\partial^{2} \xi_{\omega}}{\partial t^{2}}=0 \\
& \nabla^{2} \gamma_{\omega}-\frac{\rho}{\mu} \frac{\partial^{2} \gamma_{\omega}}{\partial t^{2}}=0
\end{aligned}
$$

On a plane wave displacing in the $\mathrm{X}$ direction, the strain in the $\mathrm{Y}$ direction must be constant in order to avoid wave spreading in this direction. Looking at Fig. 1, we see that the amplitude of vibration of mode $\theta$ must be neutralized by the amplitude of mode $\xi$. Grafting $\alpha=\pi / 2$ into Eq. (2) and equating to zero, will result

$$
\frac{\partial w}{\partial z}-\frac{\partial w}{\partial \bar{z}}=0
$$

This would be a Beltrami's equation, if the Beltrami coefficient could be equal to 1 . For $\alpha=\pi / 2$, Eq. (7) will give $\theta=\xi$ and $2 \omega=\gamma$. Actually these equations simply mean that $\frac{\partial v}{\partial y}=0$ and $\frac{\partial u}{\partial y}=0$, respectively, but an inspection of Fig. 1 allows a deeper insight. The first condition gives zero radial strain in the $\mathrm{Y}$ direction while the second condition gives zero circumferential strain in that same direction as it implies that the amplitude $\gamma / 2$ of the rotation with respect to the $\mathrm{X}$-axis, resulting from the $\gamma$ shear mode, is neutralized by the amplitude of the rotation $\omega$. Observe that although the strain in the Y direction is null, the displacement is not due to rotation $\omega$. As a result, the strain amplitudes in the radial Y direction and circumferential $\mathrm{Y}$ direction remain zero while the strain amplitude in the radial $\mathrm{X}$ direc- 
tion will be $1 / 2 \theta+1 / 2 \xi=\theta$ and the strain amplitude in the circumferential $\mathrm{X}$ direction reaches $\gamma / 2+\omega=\gamma$. It can be easily demonstrated, [12], that the condition $\theta=\xi$ gives rise to the irrotational wave equation while the condition $2 \omega=\gamma$ gives rise to the equivoluminal wave equation. Plane waves displacing in a direction forming an angle $\alpha_{0}$ with the $\mathrm{X}$-axis in a unstrained medium must comply with the condition

For an irrotational wave, as $\omega=0$, results

$$
\frac{\partial w}{\partial z}+\frac{\partial w}{\partial \bar{z}} e^{-i 2\left(\alpha_{0}+\frac{\pi}{2}\right)}=0
$$

and for an equivoluminal wave, as $\theta=0$,

$$
\begin{aligned}
& \gamma_{\theta}=\theta \sin 2 \alpha_{0} \\
& \xi_{\theta}=\theta \cos 2 \alpha_{0},
\end{aligned}
$$

$$
\begin{aligned}
& \gamma_{\omega}=2 \omega \cos 2 \alpha_{0} . \\
& \xi_{\omega}=-2 \omega \sin 2 \alpha_{0} .
\end{aligned}
$$

Equations (33) and (34) are the in-plane waveguide equations for plane waves.

\section{FINITE ROTATIONS}

The gain of area during a plane deformation is given by

$$
d A=\theta+\frac{\theta^{2}}{4}+\omega^{2}-\frac{1}{4}\left(\xi^{2}+\gamma^{2}\right)=\theta+\left|\frac{\partial w}{d z}\right|^{2}-\left|\frac{\partial w}{\partial \bar{z}}\right|^{2}=\theta+J,
$$

which is obtained from the determinant

$$
1+d A=\left[\begin{array}{cc}
1+\frac{\partial u}{\partial x} & \frac{\partial u}{\partial y} \\
\frac{\partial v}{\partial x} & 1+\frac{\partial v}{\partial y}
\end{array}\right]
$$

The symbol $J$ standsfor the Jacobian of the mapping $w(z, \bar{z})=u(x, y)+i v(x, y)$. Observing fig. 1 , it can be seen that a finite "rotation" $\omega$ has a significant influence into the change of area of a plane elastic body. In some bending and buckling problems, it is admitted to disregard squared strain terms, except $\omega^{2}$, reducing $d A$ to

$$
d A=\theta+\omega^{2}
$$

A rigid body rotation in this case can be approximated by the mathematical condition $\theta+\omega^{2}=0$. However this is not a physical description of the phenomena involved but just a consequence of the use of Cartesian coordinates. Then, for a point to describe a circular path of $\operatorname{arc} \Omega=\tan ^{-1} \omega$ it is required that the expansion produced by the mode $\omega$ be neutralized by the shrinkage $\theta=-\omega^{2}$. For the condition given by eqn. (37), the work expression in the undeformed reference frame, assumes the form

$$
U=1 / 2 \int(\lambda+\mu)\left(\theta+\omega^{2}\right)^{2}+\mu(\xi+i \gamma) \overline{(\xi+i \gamma)} e^{-i \omega} d x d y
$$


The factor $e^{-i \omega}$ is applied to the second term because the ellipses $\xi$ and $\gamma$ rotate an angle $\omega$. The first term is axis-symmetric and therefore is not affected by the rotation. The Euler equations for the functional given by Eq. (38), are

$$
\begin{array}{r}
(\lambda+\mu)\left[\frac{\partial \theta}{\partial x}-\frac{\partial}{\partial y}(\omega \theta)+\frac{\partial}{\partial x}\left(\omega^{2}\right)-3 \omega^{2} \frac{\partial \omega}{\partial y}\right]+\mu\left(\frac{\partial \xi}{\partial x}+\frac{\partial \gamma}{\partial y}\right) e^{-i \omega}=0, \\
(\lambda+\mu)\left[\frac{\partial \theta}{\partial y}+\frac{\partial}{\partial x}(\omega \theta)+\frac{\partial}{\partial y}\left(\omega^{2}\right)+3 \omega^{2} \frac{\partial \omega}{\partial x}\right]++\mu\left(\frac{\partial \gamma}{\partial x}-\frac{\partial \xi}{\partial y}\right) e^{-i \omega}=0 .
\end{array}
$$

This system of equations can be casted into the complex form

$$
(\lambda+\mu) \frac{\partial}{\partial \bar{z}}\left[(1+i \omega)\left(\theta+\omega^{2}\right)\right]+\mu \frac{\partial}{\partial z}(\xi+i \gamma) e^{-i \omega}=0
$$

Applying Green's formula in the complex form, as given by Eq. (17), the traction vector on a closed curve $C$, in the undeformed reference frame, results

$$
T=(\lambda+\mu)\left[(1+i \omega)\left(\theta+\omega^{2}\right)\right]-\mu(\xi+i \gamma) e^{-i(2 \alpha+\omega)}
$$

The traction will be represented in complex form $T=\sigma+i \tau$, with the imaginary part always rotated counterclockwise $\pi / 2$ with respect to the real part.

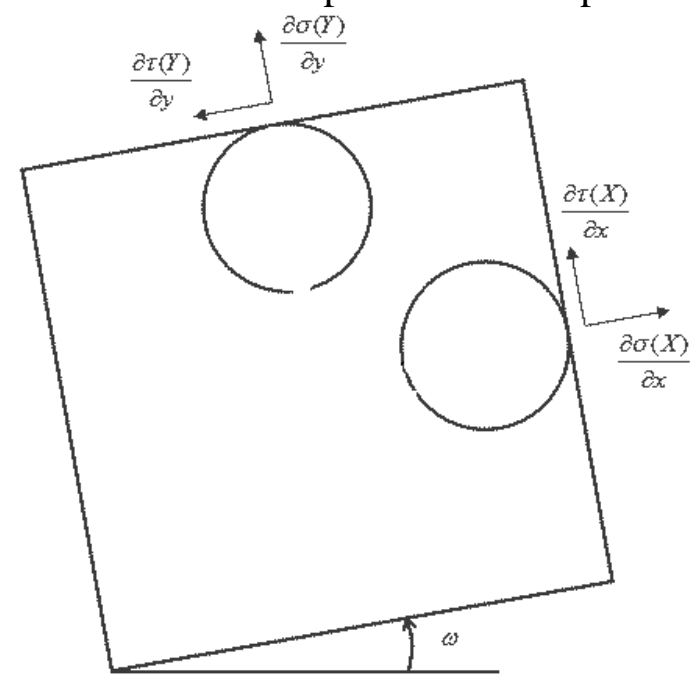

Fig.2. Traction vectors at the faces of an infinitesimal element.

Grafting $\alpha=\frac{\pi}{2}$ for the traction given by Eq. (42), at an undeformed $\mathrm{X}=$ constant plane, results

$$
\begin{aligned}
& \sigma(X)=(\lambda+\mu)\left(\theta+\omega^{2}\right)+\mu \xi \cos (\omega)-\mu \gamma \sin (\omega) \\
& \tau(X)=\omega(\lambda+\mu)\left(\theta+\omega^{2}\right)+\mu \xi \sin (\omega)+\mu \gamma \cos (\omega)
\end{aligned}
$$

Now grafting $\alpha=\pi$ for the traction at a Y=constant plane,

$$
\sigma(Y)=(\lambda+\mu)\left(\theta+\omega^{2}\right)-\mu \xi \cos (\omega)+\mu \gamma \sin (\omega)
$$




$$
\tau(Y)=\omega(\lambda+\mu)\left(\theta+\omega^{2}\right)-\mu \xi \sin (\omega)-\mu \gamma \cos (\omega)
$$

The equilibrium equations are then, (see Fig. 2):

$$
\begin{aligned}
& \frac{\partial \sigma(X)}{\partial x}-\frac{\partial \tau(Y)}{\partial y}=0 \\
& \frac{\partial \sigma(Y)}{\partial y}+\frac{\partial \tau(X)}{\partial x}=0
\end{aligned}
$$

Letting $\omega$ be finite but small and then making the following substitutions:

$$
\begin{aligned}
& (\lambda+\mu)\left(\theta+\omega^{2}\right)=\frac{\sigma_{x}+\sigma_{y}}{2} \\
& \mu \xi=\frac{\sigma_{x}-\sigma_{y}}{2} \\
& \mu \gamma=\tau_{x y}
\end{aligned}
$$

the equilibrium equations in terms of stresses, in the undeformed reference frame, become

$$
\begin{aligned}
& \frac{\partial \sigma_{x}}{\partial x}+\frac{\partial \tau_{x y}}{\partial y}-\frac{\partial}{\partial x}\left(\omega \tau_{x y}\right)-\frac{\partial}{\partial y}\left(\omega \sigma_{y}\right)=0 \\
& \frac{\partial \sigma_{y}}{\partial y}+\frac{\partial \tau_{x y}}{\partial x}+\frac{\partial}{\partial x}\left(\omega \sigma_{x}\right)+\frac{\partial}{\partial y}\left(\omega \tau_{x y}\right)=0
\end{aligned}
$$

These are exactly equilibrium equations (II.49) given by Novozhilov, [8]. The homogeneous equilibrium equations (45) can be written in the form

$$
\frac{\partial T}{\partial \bar{z}}=0
$$

Therefore, if body forces and singularities are not present, the Cauchy-Riemann equation for the tensor field $T$ is just a condition of equilibrium. Observe that for these conditions, Morera's theorem also holds true.

\section{CANONICAL FORM OF EQUILIBRIUM EQUATIONS}

Substitution of the compatibility equation (19) reduces the equilibrium equation to the following Cauchy-Riemann equation [6],

$$
\frac{\partial}{\partial \bar{z}}(\theta+i \eta 2 \omega)=0
$$

where $\eta=\frac{\mu}{\lambda+2 \mu}$. The modes $\theta$ and $\eta 2 \omega$ are hence conjugate harmonic functions. The writer succeeded in getting the equilibrium equation in another form that proved to be easily solvable. The elimination of the derivatives of $\theta$ between the compatibility equation (19) and Eq. (51) gives 


$$
\begin{aligned}
& 2 \frac{\partial \omega}{\partial x}=\frac{\lambda+2 \mu}{\lambda+\mu}\left(\frac{\partial \gamma}{\partial x}-\frac{\partial \xi}{\partial y}\right) \\
& 2 \frac{\partial \omega}{\partial y}=-\frac{\lambda+2 \mu}{\lambda+\mu}\left(\frac{\partial \xi}{\partial x}+\frac{\partial \gamma}{\partial y}\right)
\end{aligned}
$$

Now, equilibrium equations (15) give

$$
\begin{aligned}
& \frac{\partial \theta}{\partial x}=-\frac{\mu}{\lambda+\mu}\left(\frac{\partial \xi}{\partial x}+\frac{\partial \gamma}{\partial y}\right), \\
& \frac{\partial \theta}{\partial y}=-\frac{\mu}{\lambda+\mu}\left(\frac{\partial \gamma}{\partial x}-\frac{\partial \xi}{\partial y}\right)
\end{aligned}
$$

Combination of Eqs. (52) and (53) yields:

$$
2 \frac{\partial}{\partial z}(\theta+i 2 \omega)=-\chi\left[\left(\frac{\partial \xi}{\partial x}+\frac{\partial \gamma}{\partial y}\right)-i\left(\frac{\partial \gamma}{\partial x}-\frac{\partial \xi}{\partial y}\right)\right]
$$

or

$$
\frac{\partial^{2} w}{\partial z^{2}}+\chi \frac{\overline{\partial^{2} w}}{\partial z \partial \bar{z}}=0
$$

where $\chi=\frac{\lambda+3 \mu}{\lambda+\mu}$, for plane strain and $\chi=\frac{\lambda^{*}+3 \mu}{\lambda^{*}+\mu}$, for plane stress, observing that $\lambda^{*}=\frac{2 \lambda \mu}{\lambda+2 \mu}$.

\subsection{General solution}

Differentiation of Eq. (11) in $z$ results

$$
2(\lambda+\mu) \frac{\partial^{2} \theta}{\partial z \partial \bar{z}}+2 \mu \frac{\partial^{2}}{\partial z^{2}}(\xi+i \gamma)=0
$$

Since $4 \frac{\partial^{2}}{\partial z \partial \bar{z}}$ is the Laplacian operator and $\theta$ is a harmonic function, this equation reduces to

$$
2 \mu \frac{\partial^{2}}{\partial z^{2}}(\xi+i \gamma)=0
$$

and after substitution of Eq. (6), to

Integration in $z$ gives

$$
2 \frac{\partial}{\partial z}\left(\frac{\partial^{2} w}{\partial z \partial \bar{z}}\right)=0
$$

$$
\frac{\partial^{2} w}{\partial z \partial \bar{z}}=-\frac{1}{2 \mu} \overline{\phi^{\prime \prime}(z)}
$$

where $\overline{\phi^{\prime \prime}(z)}$ is the conjugate of an analytic function.

Integrating Eq. (58) in $z$, results 


$$
\frac{\partial w}{\partial \bar{z}}=\frac{1}{2}(\xi+i \gamma)=-\frac{1}{2 \mu}\left[z \overline{\phi^{\prime \prime}(z)}+\overline{\psi^{\prime}(z)}\right]
$$

Equations (54) and (58) furnish

$$
\frac{\partial^{2} w}{\partial z^{2}}=\frac{\chi}{2 \mu} \phi^{\prime \prime}(z)
$$

which after integration in $z$, results

$$
\frac{\partial w}{\partial z}=\frac{\chi}{2 \mu} \phi^{\prime}(z)+\zeta^{\prime}(\bar{z}) .
$$

Differentiation of this equation with respect to $\bar{z}$ yields

$$
\frac{\partial}{\partial \bar{z}}\left(\frac{\partial w}{d z}\right)=\zeta "(\bar{z})
$$

Comparison with Eq. (31), results

$$
\zeta "(\bar{z})=-\frac{1}{2 \mu} \overline{\phi^{\prime \prime}(z)}
$$

and hence

$$
\zeta^{\prime}(\bar{z})=-\frac{1}{2 \mu} \overline{\phi^{\prime}(z)}+c
$$

where $c$ is a complex constant. Substitution into Eq. (61) yields

$$
\frac{\partial w}{\partial z}=\frac{1}{2}(\theta+i 2 \omega)=\frac{1}{2 \mu}\left[\chi \phi^{\prime}(z)-\overline{\phi^{\prime}(z)}\right]+\frac{1}{2}\left(\theta_{0}+i 2 \omega_{0}\right) .
$$

Using Eqs. (65) and (59), the integration of the total differential, Eq. (10), gives KolosovMuskhelishvili's general solution, [4,7,9]:

$$
w=w_{0}+\frac{1}{2}\left(\theta_{0}+i 2 \omega_{0}\right) z+\frac{1}{2 \mu}\left[\chi \phi(z)-z \overline{\phi^{\prime}(z)}-\overline{\psi(z)}\right] .
$$

This linear integral can be easily verified through its derivatives in $z$ and $\bar{z}$. The addition of Eq. (65) with its conjugate gives

$$
\theta=\frac{\chi-1}{2 \mu}\left[\phi^{\prime}(z)+\overline{\phi^{\prime}(z)}\right]+\theta_{0} .
$$

The subtraction of Eq. (65) from its conjugate gives

$$
\omega=\frac{\chi+1}{2 \mu}\left[\frac{\phi^{\prime}(z)-\overline{\phi^{\prime}(z)}}{2 i}\right]+\omega_{0} .
$$

From Eqs. (67) and (65), the following Kolosov's formulas, for stresses are obtained:

$$
\begin{gathered}
\frac{\sigma_{x}+\sigma_{y}}{2}=\left[\phi^{\prime}(z)+\overline{\phi^{\prime}(z)}\right]+(\lambda+\mu) \theta_{0}, \\
\frac{\sigma_{x}-\sigma_{y}}{2}+i \tau_{x y}=-\left[z \overline{\phi^{\prime \prime}(z)}+\overline{\psi^{\prime}(z)}\right] .
\end{gathered}
$$

The traction $T=\sigma+i \tau$, see Fig.2, in terms of analytic functions, results

$$
T=\phi^{\prime}(z)+\overline{\phi^{\prime}(z)}+\left[z \overline{\phi^{\prime \prime}(z)}+\overline{\psi^{\prime}(z)}\right] e^{-2 i \alpha}+(\lambda+\mu) \theta_{0}
$$


The integration of the traction over any boundary $C$, enclosing a domain, gives the resultant of all forces acting in that domain

$$
X+i Y=-i \oint_{C}\left[\phi^{\prime}(t)+\overline{\phi^{\prime}(t)}\right] d t+\left[t \overline{\phi^{\prime \prime}(t)}+\overline{\psi^{\prime}(t)}\right] d \bar{t}
$$

or

$$
X+i Y=\phi(t)+t \overline{\phi^{\prime}(t)}+\overline{\psi(t)}+C
$$

where $C$ is a complex constant.

\section{FINITE ROTATION WAVES IN INCOMPRESSIBLE MEDIA}

Let us look for an exact equation of a plane equivoluminal wave. Accordingly with Eq. (35) we must have

$$
\theta+\frac{\theta^{2}}{4}+\omega^{2}-\frac{1}{4}\left(\xi^{2}+\gamma^{2}\right)=0
$$

with $\theta=0$. Hence besides the compatibility equations (12) and (13), the following condition must be accomplished:

$$
\omega^{2}=\frac{1}{4}\left(\xi^{2}+\gamma^{2}\right)
$$

and in addition, the equilibrium equation (15), in the undeformed reference frame, reduces to

$$
\begin{aligned}
& \frac{\partial \xi}{\partial x}+\frac{\partial \gamma}{\partial y}-\frac{\rho}{\mu} \frac{\partial^{2} u}{\partial t^{2}}=0 \\
& \frac{\partial \gamma}{\partial x}-\frac{\partial \xi}{\partial y}-\frac{\rho}{\mu} \frac{\partial^{2} v}{\partial t^{2}}=0
\end{aligned}
$$

From $\theta=0$ and Eq. (75) we get

$$
\frac{\partial v}{\partial y}=-\frac{\partial u}{\partial x}, \quad \frac{\partial v}{\partial x}=-\frac{\left(\partial u / \partial x^{2}\right.}{\partial u / \partial y}, \frac{\partial u}{\partial y}=-\frac{\left(\partial v / \partial y^{2}\right.}{\partial v / \partial x}
$$

Substitution of these relations into Eq. (76), results in the following uncoupled nonlinear partial differential equations:

$$
\begin{aligned}
& 2 \frac{\partial^{2} u}{\partial x^{2}}+\frac{\partial^{2} u}{\partial y^{2}}\left[1+\left(\frac{\partial u / \partial x}{\partial u / \partial y}\right)^{2}\right]-2 \frac{\partial^{2} u}{\partial x \partial y}\left(\frac{\partial u / \partial x}{\partial u / \partial y}\right)-\frac{\rho}{\mu} \frac{\partial^{2} u}{\partial t^{2}}=0 \\
& 2 \frac{\partial^{2} v}{\partial y^{2}}+\frac{\partial^{2} v}{\partial x^{2}}\left[1+\left(\frac{\partial v / \partial y}{\partial v / \partial x}\right)^{2}\right]-2 \frac{\partial^{2} v}{\partial x \partial y}\left(\frac{\partial v / \partial y}{\partial v / \partial x}\right)-\frac{\rho}{\mu} \frac{\partial^{2} v}{\partial t^{2}}=0
\end{aligned}
$$

The solution of these equations will probably require procedures of computational analysis. 


\section{REFERENCES}

[1] Achenbach, J. D., Wave Propagation In Elastic Solids, Elsevier, pp. 187-194, 1999.

[2] Courant R. \& Hilbert, D., Methods of Mathematical Physics, Vol. II, Wiley: New York, pp. 350-351, 1989.

[3] Elmore, W. C. and Heald, M. A., Physics of Waves, Dover Publications, 1985.

[4] England, A. H., Complex Variable Methods in Elasticity, Wiley-Interscience, pp. 28-49, 1971.

[5] Graff, K.L., Wave Motion in Elastic Solids, Oxford. The Clarendon Press, 1975.

[6] Love, A.E.H., A Treatise on the Mathematical Theory of Elasticity, Cambridge University Press, pp. 204-220, 1927.

[7] Muskhelishvili, N. I., Some Basic Problems of the Theory of Elasticity, Noordhoff, Groningen, 1963.

[8] Novozhilov, V.V., Foundations of the Nonlinear Theory of Elasticity, Dover Publications, Inc.: Mineola, New York, pp. 83-84, 1999.

[9] Sokolnikoff, I. S., Mathematical Theory of Elasticity, McGraw-Hill, 1956.

[10] Kotchergenko, I.D., The Areolar Strain Concept, IMECE2008 ASME International Mechanical Engineering Congress and Exposition, Boston, October 2008.

[11] Kotchergenko, I.D., Kolosov-Mushkhelishvili Formulas Revisited, $11^{\text {th }}$ International Conference on Fracture, Turin, March 2005.

[12] Kotchergenko, I.D., Applications of Generalized Analytic Functions to Elasticity, CMM2005-Computer Methods in Mechanics, Polish Academy of Sciences, June 2005.

[13] Kotchergenko, I.D., The Areolar Strain Concept Applied to Elasticity, WIT Transactions on Modelling and Simulation, Vol. 46, 2007, WIT Press, (free).

[14] Mitrinovic, D.S., Keckic, J.D., From the History of Nonanalitic Functions, Série:

Mathématiques e Physique, No. 274-371, Publications de La Faculté D’Electrotechnique de L’Université à Belgrade, 1969, (free). 